\title{
CURVA DE PARÂMETROS SANGUÍNEOS E DE PESO EM SPHENISCUS MAGELLANICUS (FOSTER, 1781) (AVES: SPHENISCIFORMES) EM REABILITAÇÃO COM ASPERGILOSE
}

\author{
(Curve of blood and weight parameters in Spheniscus magellanicus (Foster, \\ 1781)(Birds: Sphenisciformes) in Rehabilitation with Aspergillosis)
}

\author{
Aryse Martins Melo ${ }^{1}$, Melissa Orzechowski Xavier, Rodolfo Pinho da Silva Filho, Angela \\ Leitzke Cabana, Mário Carlos Araújo Meireles \\ ${ }^{1}$ Correspondência: arysemartins@gmail.com
}

\begin{abstract}
RESUMO: Parâmetros sanguíneos básicos e peso corpóreo são rotineiramente utilizados para monitoramento do estado geral de Pinguins-de-Magalhães em reabilitação, no entanto, estudos mostrando o perfil de variação destes parâmetros durante o desenvolvimento da aspergilose nestes animais não são conhecidos. Neste sentido, este trabalho objetivou determinar curva de peso, de hematócrito $(\mathrm{Ht})$ e de proteínas plasmáticas totais (PPT) em pinguins com aspergilose. O estudo do tipo caso-controle retrospectivo foi realizado com pinguins em reabilitação no sul do Brasil, sendo o grupo caso composto por pinguins com aspergilose, e o grupo controle por pinguins sadios. Para a determinação das curvas, foram coletados dados de amostras sequenciais, realizadas em média a cada sete dias, durante um período máximo de 81 dias, sendo estes submetidos a análise de variância (ANOVA) com teste post-hoc de Bonferroni. Ao todo, 140 animais foram estudados ( $50 \%$ casos e $50 \%$ controles). Pinguins com aspergilose diferiram significativamente do grupo controle em todos os parâmetros analisados, apresentando ganho de peso somente nas três primeiras coletas, com estabilização ou perda de peso nas coletas posteriores até seu desfecho, bem como declínio progressivo dos valores de $\mathrm{Ht}$, os quais mantiveram-se abaixo do valor de referência para a espécie desde a terceira até a nona e última coleta, e aumento progressivo dos valores de PPT ao longo das coletas, com diferença significativa a partir da sexta coleta em relação ao grupo controle. $O$ estabelecimento desse perfil em pinguins com aspergilose pode servir como indicativo de mau prognóstico destes animais em cativeiro, e como parâmetro para início de terapia preemptiva para aspergilose ou de investigação diagnóstica mais específica.
\end{abstract}

Palavras-chave: Aspergillus; hematócrito; proteínas plasmáticas totais; Sphenisciformes

ABSTRACT: Basic blood parameters and body weight are routinely used to monitor the general condition of Magellanic penguins in rehabilitation, however, studies showing the variation profile of these parameters during the development of aspergillosis in these animals are not known. Thus, this study aimed to determine the curve of weight, hematocrit $(\mathrm{Ht})$ and total plasma proteins (PPT) in penguins with aspergillosis. The study of retrospective case-control was carried out with penguins in rehabilitation in southern Brazil, and the case group was composed by penguins with aspergillosis, and the control group by healthy penguins. For the determination of the curves, data were collected from sequential samples, performed on average every seven days for a maximum period of 81 days, which underwent analysis of variance (ANOVA) with post-hoc Bonferroni. A total of 140 animals were studied (cases 50\% and 50\% control). Penguins with aspergillosis differed significantly from the control group in all parameters, with weight gain only in the first three collections, with stabilization or weight loss in later collections until its upshot, and progressive decline of $\mathrm{Ht}$ values, which were maintained below the reference value for the specie from the third to the ninth and final collection, and progressive increase of PPT values during the experimental period, with a significant difference since the sixth collection in the control group. The establishment of this profile in penguins with aspergillosis can serve as an indicator of poor prognosis of these animals in captivity, and as a parameter for early preemptive therapy for aspergillosis or more specific diagnosis.

Key Words: Aspergillus; hematocrit; total plasma protein; Sphenisciformes

Recebido em 23/03/2015

Aprovado em 03/06/2017 


\section{INTRODUÇÃO}

A aspergilose é uma doença fúngica, não contagiosa, considerada uma das principais causas de óbito em pinguins em reabilitação. A infecção ocorre principalmente por via aérea, a partir da inalação de propágulos de Aspergillus spp. os quais estão dispersos em todos os ambientes. O trato respiratório inferior é o principal sistema acometido, embora muitas vezes ocorra disseminação via hematógena ou por contiguidade para outros órgãos, geralmente culminando na morte do indivíduo infectado (Carrasco et al., 2001; Xavier et al, 2007; 2011).

O diagnóstico dessa doença em pinguins em cativeiro ainda é estabelecido principalmente por exames post mortem, a partir da visualização na necropsia de granulomas e/ou colônias fúngicas em sacos aéreos, associada a exames micológicos e histopatológico (Carrasco et al., 2001; Xavier et al., 2011). Isto ocorre principalmente devido ao fato destas aves infectadas comumente apresentarem sinais clínicos inespecíficos e tardios, ou até mesmo morte súbita (AbundisSantamaria, 2003; Beytut et al., 2004; Xavier et al., 2007).

Embora a doença seja descrita em pinguins em cativeiro desde a década de 40 (Ainsworth; Rewell, 1949; Flach et al., 1990; Khan et al., 1977), ainda hoje não existem métodos eficazes e acessíveis para diagnóstico in vivo da aspergilose nestes animais. Estudos recentes tem demonstrado o valor da avaliação de valores de hematócrito e de proteínas plasmáticas totais, albumina e globulinas, no diagnóstico da aspergilose em diversas espécies de aves (Black et al., 2013; Cray et al., 2009, Davidow et al., 1997; Graczyk et al., 1998).

Esses exames hematológicos básicos, como a determinação do hematócrito e das proteínas plasmáticas totais, em associação com ganho de peso são rotineiramente utilizados para monitoramento do estado geral de Spheniscus magellanicus (pinguins-deMagalhães) durante 0 processo de reabilitação, especialmente devido ao baixo custo e alta acessibilidade. Valores de referência destes parâmetros, estabelecidos por protocolos, são utilizados como critérios para liberação, caracterizando os pinguins aptos à reintrodução na natureza (Ruoppolo et al., 2004; Martins et al., 2015). No entanto, o comportamento destes parâmetros sanguíneos básicos e das medidas de ganho de peso em pinguins-deMagalhães com aspergilose em cativeiro ainda não foi descrito.

Considerando que

conhecimento desses dados, os quais são rotineiramente coletados em Centros de reabilitação, pode contribuir com a identificação de animais potencialmente infectados por Aspergillus spp., este trabalho objetivou avaliar a curva de peso, de ganho de peso e de parâmetros sanguíneos como hematócrito e PPT em $S$. magellanicus que morreram por aspergilose durante a reabilitação.

\section{MATERIAL E MÉTODOS}

O estudo do tipo caso-controle retrospectivo foi realizado no Centro de Recuperação de Animais Marinhos (CRAM-FURG), anexo ao Museu Oceanográfico Prof. Eliézer de Carvalho Rios, da Universidade Federal do Rio Grande (FURG), no Rio Grande do Sul, Brasil. As informações foram coletadas do banco de dados do CRAM-FURG referentes a um período de cinco anos (janeiro de 2008 a dezembro 2012).

Foram caracterizados como casos e incluídos no estudo todos os pinguinsde-Magalhães que desenvolveram 
aspergilose durante a reabilitação. O diagnóstico destes casos foi confirmado por exames post-mortem a partir de achados macroscópicos em necropsia com confirmação laboratorial por isolamento de Aspergillus sp. em cultivo microbiológico associado a confirmação de invasão fúngica tecidual no exame histopatológico. O grupo controle foi selecionado aleatoriamente incluindo o mesmo número de animais do grupo caso, e composto por pinguins que passaram pelo mesmo processo de recuperação e não apresentaram nenhuma intercorrência nem doença oportunista, tendo sido liberados hígidos ao habitat natural.

Os dados foram coletados inicialmente uma vez por semana por aproximadamente quatro semanas, período no qual os animais ainda estavam passando pela estabilização do seu quadro sanitário, necessitando dessa forma, acompanhamento maior dos parâmetros básicos de saúde. Após aproximadamente quatro semanas, as coletas ocorreram quinzenalmente ou mensalmente, dependendo da rotina do centro e do estado sanitário apresentado pelos animais. Esse aumento no intervalo entre as coletas ocorreu principalmente com a finalidade de diminuir o estresse dos animais já que, em sua maioria, depois de quatro semanas de reabilitação os animais já apresentavam um quadro estável dos parâmetros coletados. A periodicidade dos dados coletados seguiu a rotina de avaliação dos animais realizada no CRAM-FURG, sendo utilizada a mediana de todos os animais de cada grupo para determinação do tempo entre cada coleta.

Valores de hematócrito, PPT e peso corpóreo foram analisados em todos os pinguins, além disso, foi calculado o ganho de peso individual entre cada coleta. Estes dados foram acompanhados com coletas desde a chegada do animal no CRAM-FURG até o desfecho dos animais do grupo caso, óbito por aspergilose. Para a determinação das curvas de cada parâmetro, foi realizada a análise de variância (ANOVA) com teste post-hoc de Bonferroni para comparação entre os grupos. A análise estatística foi realizada com auxílio do programa Stata, versão 11.1.

\section{RESULTADOS E DISCUSSÃO}

Um total de 140 pinguins foi incluído no estudo, 70 (50\%) no grupo controle e 70 (50\%) no grupo caso aspergilose. O período de avaliação dos parâmetros correspondeu a 81 dias, contemplando nove coletas de dados. $A$ mediana de tempo decorrido entre cada uma das primeiras quatro coletas de dados foi de sete dias, aumentando para oito dias entre a quarta e quinta coleta, nove dias entre a sexta e sétima coleta, e 17 e 19 dias entre a sétima e oitava e, oitava e nona coletas, respectivamente.

Os resultados da mediana de tempo de coleta refletem a rotina de manejo desenvolvida no Centro de Recuperação de Animais Marinhos CRAM-FURG, de forma que, preferencialmente, os exames de acompanhamento dos animais ocorrem semanalmente nas primeiras quatro semanas, passando para quinzenalmente, podendo chegar a mensalmente de acordo com o tempo de cativeiro dos animais.

Perdas foram ocorrendo com o decorrer do período de acompanhamento dos animais, tanto por óbito devido a aspergilose (grupo caso) quanto por liberação (grupo controle), resultando em um número total de onze pinguins na última avaliação (nona coleta) (Tabela 1). Em adição, a variação no número de animais analisados entre as coletas pode ser atribuída à perda de dados de algumas coletas, as quais não 
tiveram os resultados repassados para o banco de dados.

\begin{tabular}{|c|c|c|c|}
\hline \multicolumn{4}{|c|}{$\begin{array}{l}\text { Tabela - } 1 \text { Número de pinguins incluídos nas análises em cada } \\
\text { coleta de acordo com o grupo pertencente: caso (animais que } \\
\text { foram a óbito por aspergilose) ou controle (animais liberados). }\end{array}$} \\
\hline Coleta & Grupo controle & Grupo caso & Total \\
\hline 1 & 69 & 69 & 138 \\
\hline 2 & 69 & 57 & 126 \\
\hline 3 & 70 & 54 & 124 \\
\hline 4 & 69 & 49 & 118 \\
\hline 5 & 62 & 38 & 100 \\
\hline 6 & 45 & 25 & 70 \\
\hline 7 & 30 & 20 & 50 \\
\hline 8 & 15 & 7 & 22 \\
\hline 9 & 9 & 2 & 11 \\
\hline
\end{tabular}

As perdas de animais no grupo caso ao longo das coletas eram esperadas na medida em que a doença em pinguins é fatal, uma vez que o tratamento ainda é inviável devido à ausência de exames efetivos para o diagnóstico precoce da aspergilose em pinguins (Jones; Orosz, 2000; Xavier et al., 2007). Da mesma forma, a redução do número de animais no grupo controle é atribuída ao fato de que os animais já recuperados devem ser reintroduzidos ao habitat natural no menor período de tempo possível, a fim de evitar o aparecimento das doenças secundárias ao cativeiro, as quais podem levar o animal à óbito, como a malária aviária e a pododermatite, além da própria aspergilose (Russel et al., 2003; SilvaFilho; Ruoppolo; 2014).

Em relação ao peso corpóreo dos pinguins, o valor médio na chegada dos animais, ou seja, na primeira coleta foi de $2.747 \mathrm{~g}$ no grupo caso e $2.875 \mathrm{~g}$ no grupo controle, sem diferença significativa $(P=0,182)$. Os animais com aspergilose tiveram pouco aumento de peso ao longo da reabilitação e, quando comparados com os animais sadios, a partir da segunda coleta até a oitava, excetuando-se a sexta coleta a média de peso desse grupo foi significativamente menor do que a do grupo controle (Figura 1).

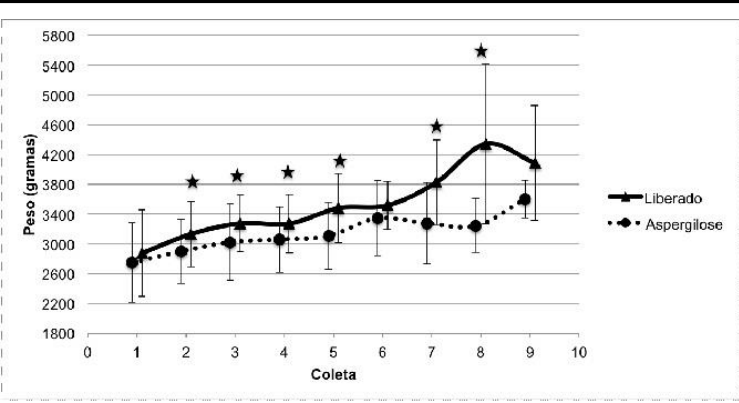

Figura 1 - Curva de peso corpóreo médio dos pinguins ao longo da reabilitação, demonstrando diferença significativa entre o grupo caso (aspergilose) e o grupo controle (animais liberados) em algumas coletas ${ }^{\star} \mathrm{P}<0,05$.

Embora animais do grupo caso também tenham apresentado um aumento da média de peso durante a reabilitação, este foi menos expressivo quando comparado com o grupo controle, sendo o ganho de peso máximo entre coletas em animais com aspergilose de $548 \mathrm{~g}$, enquanto que em animais do grupo controle foi de $1.259 \mathrm{~g}$.

Em adição, no grupo de animais com aspergilose, em cinco dos oito intervalos de coleta a média de peso dos animais manteve-se estável ou reduziu, enquanto que no grupo controle, ganho de peso foi observado em praticamente todos os intervalos de coleta, havendo perda de peso somente entre a penúltima e a última coleta (Figura 2). A manutenção da tendência a estabilidade ou redução do peso nos animais com aspergilose ocorreu a partir do terceiro intervalo de coleta, que correspondeu a 21 dias de cativeiro.

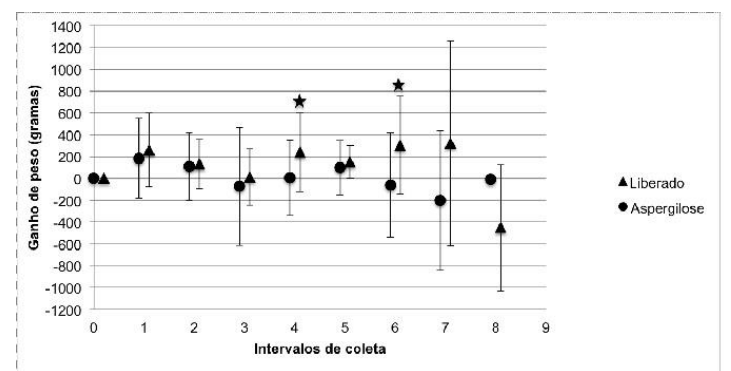

Figura 2 - Média de ganho de peso entre as coletas realizadas ao longo da reabilitação de pinguins, comparando o grupo caso (aspergilose) e o grupo controle (animais liberados) ${ }^{\star} \mathrm{P}<0,05$.

Segundo Crissey et al. (2002), pinguins se alimentam de mais de uma variedade de peixe quando estão em vida livre, o que proporciona uma dieta 
rica em energia e vitaminas. Dessa forma, devem receber uma alimentação balanceada em cativeiro com reposição de vitaminas, sobretudo tiamina e vitamina $E$. Durante o período de reabilitação no CRAM-FURG, todos os pinguins recebem alimentação seguindo o protocolo de Ruoppolo et al. (2004), portanto, a oferta de alimento não pode ser considerada um fator responsável pela diferença de aumento de peso entre os dois grupos, já que ambos receberam o mesmo tratamento.

A literatura cita regurgitação, inapetência e anorexia como sinais clínicos da aspergilose em aves marinhas, (Risi et al., 2003; Tell, 2005) incluindo pinguins (Nakeeb et al., 1981; Xavier et al., 2007). De fato, alguns desses sinais já foram descritos em animais que chegaram ao CRAM-FURG e morreram por aspergilose durante a reabilitação. A apresentação de inapetência e regurgitação pode ser considerada um fator contribuinte para o menor ganho de peso e até mesmo a perda de peso do grupo caso em relação ao grupo controle.

Em relação ao hematócrito $(\mathrm{Ht}) \mathrm{o}$ valor médio na chegada dos animais ao CRAM-FURG, correspondente a primeira coleta, foi de $38 \%$ e $44 \%$ nos grupos caso e controle, respectivamente. No grupo de pinguins com aspergilose foi detectado um declínio progressivo na curva dos valores de $\mathrm{Ht}$ ao longo do período de reabilitação, os quais permaneceram abaixo do limite inferior de referência para a espécie $(42 \% \pm 4 \%)$ (Hawkey et al., 1989) desde o $21^{\circ}$ dia de cativeiro, correspondendo a terceira coleta. Este perfil diferiu significativamente do grupo controle, no qual a média de $\mathrm{Ht}$ tendeu a baixar entre a primeira e segunda coleta, estabilizando-se a partir da segunda coleta (14 dias) e mantendo-se dentro do padrão de referência em todas as coletas (Figura 3).

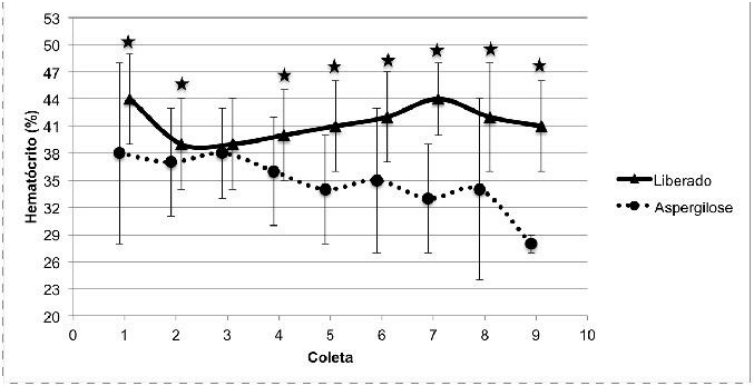

Figura 3 - Média de hematócrito dos pinguins ao longo da reabilitação, comparando $\circ$ grupo caso (aspergilose) e o grupo controle (animais liberados) ${ }^{\star} P<0,05$

A queda progressiva da média dos valores de $\mathrm{Ht}$ da terceira coleta até o desfecho no grupo caso ocorre provavelmente devido à produção e liberação de hemolisinas pelo Aspergillus sp. durante a infecção, as quais são responsáveis pela lise das hemácias e consequente anemia, o que já foi relatado para aves com aspergilose, principalmente na forma crônica da doença (Beernaert et al., 2010; Jones; Orosz, 2000).

Estudo recente realizado por Black et al. (2013) também demonstrou queda do hematócrito em Eudromia elegans (martinetas) com aspergilose quando comparadas com indivíduos saudáveis. Em contrapartida, diferença significativa nos valores de $\mathrm{Ht}$ não foi encontrada em Anas platyrhynchos (pato-real) e Tadorna cana (tardona-do-cabo) infectadas por Aspergillus quando comparados com indivíduos saudáveis (Graczyk et al. 1998), indicando que este parâmetro pode variar de acordo com a espécie de ave, provavelmente devido a diferença de suscetibilidade a infecção por Aspergillus spp. (Asakura et al., 1962).

A primeira coleta de dados de PPT demonstrou um valor médio similar, de $6,4 \mathrm{~g} / \mathrm{dL}$ e $6 \mathrm{~g} / \mathrm{dL}$, entre os grupos caso e controle, respectivamente. No entanto, na análise da curva, o grupo com aspergilose apresentou aumento progressivo desses valores durante toda a reabilitação, alcançando um valor médio de $10,3 \mathrm{~g} / \mathrm{dL}$ na nona coleta, diferindo significativamente do grupo 
controle no qual a partir da sexta coleta os valores permaneceram praticamente estabilizados em 7,9 a $8 \mathrm{~g} / \mathrm{dL}$ (Figura 4).

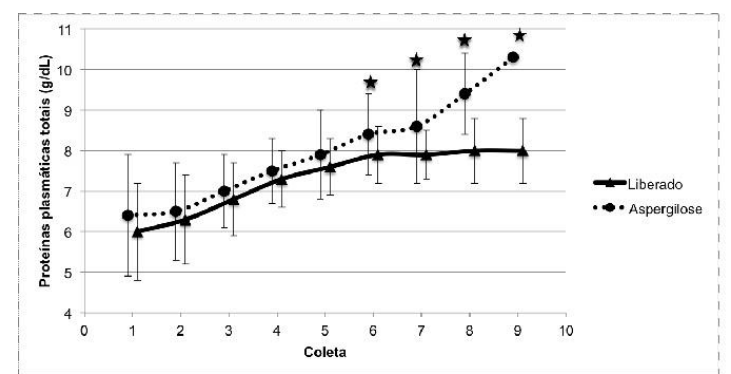

Figura 4 - Média de proteínas plasmáticas totais dos pinguins ao longo da reabilitação, comparando o grupo caso (aspergilose) e o grupo controle (animais liberados) ${ }^{*} \mathrm{P}<0,05$

No grupo caso, as PPT continuaram em elevação durante a reabilitação, 0 que pode estar relacionado a produção de imunoglobulinas pelo hospedeiro, que se caracterizam como um grande aliado no combate à aspergilose em organismos imunocompetentes (Beernaert et al., 2010). O aumento de imunoglobulinas durante um quadro de aspergilose já foi comprovado em martinetas (Black et al., 2013) e em diversas outras espécies de aves (Cray et al., 2009). De fato, testes sorológicos buscando detectar anticorpos específicos para a doença são indicados como métodos diagnósticos da aspergilose em aves, comprovando o estímulo da resposta humoral durante um quadro infeccioso por Aspergillus spp (Graczyk et al., 1998; Tell, 2005).

$O$ incremento significativo nos valores de PPT dos pinguins com aspergilose foi observado cerca de 15 dias após o início da alteração significativa na curva de $\mathrm{Ht}$ e de ganho de peso desses animais, que ocorreu a partir da terceira coleta de dados. Esse dado contribui com a suposição de que o aumento nos valores de PPT encontrado no grupo caso do presente estudo está relacionado a um aumento das globulinas, assim como descrito por Black et al. (2013). E, pode ser atribuído à produção de anticorpos específicos para a infecção, os quais geralmente são detectados em alta concentração na circulação sanguínea somente a partir de uma a duas semanas do decorrer do processo infeccioso (Shoham; Levitz, 2005).

Protocolos de reabilitação indicam a utilização da avaliação de valores de hematócrito, proteínas plasmáticas totais e medidas de peso como critérios para liberação de pinguins-deMagalhães (Ruoppolo et al., 2004). Neste trabalho observa-se que estes parâmetros também podem ser utilizados como indicadores de animais potencialmente infectados, uma vez que o resultado das curvas geradas a partir das análises mostraram diferenças pontuais no grupo de pinguins com aspergilose quando comparado com animais sadios.

\section{CONCLUSÃO}

Os Spheniscus magellanicus com aspergilose durante a reabilitação apresentam um perfil de menor ganho de peso corpóreo, declínio progressivo da curva do hematócrito, e aumento significativo das proteínas plasmáticas totais após 15 dias do início da queda do $\mathrm{Ht}$ e do ganho de peso neste grupo, quando comparados com animais hígidos. $O$ estabelecimento desse perfil em pinguins com aspergilose pode servir como indicativo de mau prognóstico destes animais em cativeiro, e como parâmetro para início de terapia preemptiva ou de investigação diagnóstica mais específica.

\section{NOTAS INFORMATIVAS}

Reservado ao parecer CEUA.

\section{REFERÊNCIAS}

ABUNDIS-SANTAMARIA, E. Aspergillosis in birds of prey, 2003. Disponível 
http://www.aspergillus.org.uk. Acesso em: 23/mar/2005.

ASAKURA, S.; NAKAGAWA, S.; MASUI $M$. Immunological Studies of Aspergillosis. In: Birds. Research Institute for Microbial Diseases, v. 18, n. 7, p. $249-256,1962$.

AINSWORTH, G.C.; REWELL, R.E. The incidence of aspergillosis in captive wild birds. Journal of Comparative Pathology and Therapeutics, v.59, p.213-224, 1949.

BEERNAERT, L. A.; PASMANS, F.; VAN WAEYENBERGHE, $\mathrm{L}$. et al. Aspergillus infections in birds: a review. Avian Pathology, v.39, n. 5, p. 325331, 2010.

BEYTUT, E.; ÖZCAN, K; ERGINSOY, S. Immunohistochemical detection of fungal elements in the tissues of goslings with pulmonary and systemic aspergillosis. Acta Veterinaria Hungarica, v.52, n.1, p.71-84, 2004.

BLACK, P. A.; MACEK, M.; TIEBER, A. et al. Reference values for hematology, plasma biochemical analysis, plasma protein electrophoresis and Aspergillus serology in elegante-crested tinamou (Eudromia elegans). Journal of Avian Medicine and Surgery, v. 27, n. 1, p.16, 2013.

CARRASCO, L.; LIMA Jr., J. S.; HALFEN, D. C. et al. Systemic Aspergillosis in an Oiled Magallanic Penguin (Spheniscus magellanicus). Journal of Veterinary Medicine, v.48, p.551-554, 2001.

CRAY, C.; WATSON, T.; RODRIGUEZ, $M$. et al. Application of galactomannan analysis and protein electrophoresis in the diagnosis of aspergillosis in avian species. Journal of Zoo and Wildlife Medicine, v. 40, n.1, p. 64-70, 2009.

CRISSEY, S.; MCGILL, P.; SLIFKA, K. [2002]. Penguins: Nutrition and Dietary Husbandry. Disponível em: $<$ <ttp://www.nagonline.net/husbandry/Di
ets\%20pdf/Penguin\%20Nutrition.pdf > Acesso em 15/10/2011.

DAVIDOW, E. B.; JOSLIN, J.; COLLINS, D. M. et al. Serial Aspergillus antibody levels and serum protein electrophoresis as a diagnostic and treatment monitoring technique in Humboldt penguins (Spheniscus humboldti). Proceedings American Association of Zoo Veterinarians, p. 31-35, 1997.

FLACH, E.J.; STEVENSON, M.F.; HENDERSON, G.M. Aspergillosis in Gentoo penguins (Pygoscelis papua) at Edinburgh Zoo, 1964-1988. Veterinary Record, v.126, n.4, p.81-85, 1990.

GRACZYK, T.K.; CRANFIELD, M.R.; KLEIN, P.N. Value of antigen and antibody detection, and blood evaluation parameters in diagnosis of avian invasive Aspergillosis. Mycopathologia, v.140, p.121-127, 1998.

HAWKEY, C. M.; HORSLEY, D. T.; KEYMER, I. F. Haematology of wild penguins (spenisciformes) in the Falkland Islands. Avian Pathology, v.18, p.495-502, 1989.

JONES, M. P.; OROSZ, S. E. The diagnosis of aspergillosis in birds. Seminars in Avian and Exotic Pet Medicine, v. 9, n. 2, p. 52-58, 2000.

KHAN Z.U, PAL M, PALIWAL D.K. et al. Aspergillosis in imported penguins. Sabouraudia, v. 15, p. 43-45, 1977.

MARTINS, A. M.; SIIVA-FILHO; R. P.; XAVIER, M. O. et al. Blood parameters and measurements of weight in the rehabilitation of Magellanic penguins (Spheniscus magellanicus, Foster 1781). Arquivo Brasileiro de Medicina Veterinária e Zootecnia, v. 67, n.1, p. 125-130, 2015.

NAKEEB, S. M.; BABUS, S. B.; CLIFTON, A. Y. Aspergillosis in the Peruvian penguin (Spheniscus humboldti). Journal of Zoo Animal Medicine, v.12, p.51-54, 1981. 
RISI, E.; THARY, V.; ARNÉ, P. et al. Aspergillosis of Seabirds in Captivity. In: INTERNATIONAL EFFECTS OF OIL ON WILDLIFE CONFERENCE, 7., 2003, Hamburg. Proceedings of the $7^{\text {th }}$ International Effects of Oil on Wildlife Conference Hamburg, Germany, 2003 p. 14. -16 .

RUOPPOLO,V.; ADORNES, A. C.; NASCIMENTO, A. C. et al. Reabilitação de pinguins afetados por petróleo. Clínica Veterinária, ano IX, n.51, p.7883, 2004.

RUSSEL, M.; HOLCOMB, J.; BERKNER, A. 30-Years of Oiled Wildlife Response Statistics. Proceedings of the $7^{\text {th }}$ International Effects of Oil and Wildlife Conference Hamburg, Germany, p.1-18, 2003.

SHOHAM, S.; LEVITZ, S.M..The immune response to fungal infections. British Journal of Haematology, v. 129, p. 569-582, 2005.

SILVA-FILHO, R. P.; RUOPPOLO, V. Sphenisciformes (Pinguim). In: CUBAS, Z.S.; SILVA, J.C.R.; CATÂO-DIAS, J.L.: Tratado de Animais SelvagensMedicina Veterinária. 2.ed. São Paulo, SP: Roca, 2014. v.1 p.384-416.

TELL, L. A. Aspergillosis in mammals and birds: impact on veterinary medicine. Medical Mycology, v.43, p.71-73, 2005.

XAVIER M.O, SOARES M.P, CABANA A.L. et al. Clinical and pathological findings of aspergillosis in magellanic penguins (Spheniscus magellanicus). Ciência Animal Brasileira, v.12, n. 3, p. 520-524, 2011.

XAVIER, M.O.; SOARES, M. P. ; MEINERZ, A. R. et al. Aspergillosis: a limiting factor during recovery of captive magellanic penguins. Brazilian Journal of Microbiology, v.38, p.480-484, 2007. 